\title{
Challenges and chances by involving community interpreters in the regular health care of pregnant women and young mothers from third countries with language barriers. A qualitative study within the project RundUm - Transcultural network in the care of pregnancy and birth
}

\author{
Herausforderungen und Chancen bei der Hinzuziehung von \\ Sprach- und Integrationsmittlerinnen in die Regelversorgung \\ von Schwangeren und jungen Müttern aus Drittstaaten. Eine \\ qualitative Erhebung im Rahmen des Projektes RundUm - \\ Transkulturelles Netzwerk zur Begleitung bei Schwangerschaft \\ und Geburt
}

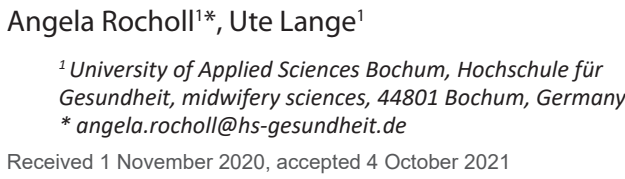

\begin{abstract}
Introduction: For linguistic understanding and the participation in the social and health care structures for people with language barriers and a migration or refugee background, the area of activity of community interpreters emerged in delimitation to standard interpreters. Belonging to the RundUm project, the community interpreters at SprInt in Essen accompany pregnant women and young mothers to regular appointments. The intention of this article is to provide information on the opportunities and challenges within obstetric care from the perspective of community interpreters.

Method: Qualitative expert interviews were conducted with 14 employees from the community interpreter service SprInt in Essen. Interviews were analyzed using thematic analysis.

Results: Challenges arose regarding the access to the clients, the cooperation with the professionals and relationship between professionals and their patients/women. Among these lacking language skills, the teaching of intercultural differences and the prevailing uncertainty of professionals dealing with language mediation influencing their work. Additionally, differently understood roles on the part of the Sprlnters offer opportunities and conflicts for further cooperation in care network of pregnant women and young mothers.

Discussion: The importance of community interpreting for the appropriate obstetric care of women with language problems offers opportunities for a public discussion on the job profile and the generation of new fields of activity, representing a bridge and navigation function. This requires secure financing options for the involvement of the community interpreters in the care of pregnant women and young mothers and an implementation of interprofessional network structures.
\end{abstract}

\begin{abstract}
Einleitung: Zur sprachlichen Verständigung und Partizipation an den Versorgungsstrukturen des Sozial- und Gesundheitswesens bei Menschen mit Sprachbarrieren und einem Migrations- oder Fluchthintergrund entwickelte sich der Tätigkeitsbereich der Sprach- und Integrationsmittler/in in Abgrenzung zum/zur Dolmetscher/-in. Im Rahmen des Forschungsprojektes RundUm begleiteten Sprach- und Integrationsmittlerinnen des Sprachmittlerdienstes Sprlnt in Essen Schwangere und junge Mütter zu Terminen der Regelversorgung im Gesundheitswesen. Die Chancen und Herausforderungen innerhalb der geburtshilflichen Versorgung aus Sicht der Sprach- und Integrationsmittlerinnen sind Gegenstand der vorliegenden Studie.

Methode: Im Rahmen von qualitativen Experteninterviews wurden 14 Sprach- und Integrationsmittelnde des Sprachmittlerdienstes Sprlnt in Essen befragt. Die Transkripte wurden inhaltsanalytisch ausgewertet.

Ergebnisse: Herausforderungen zeigen sich in der Kontaktaufnahme zu den Klientinnen und in Aspekten der Zusammenarbeit mit professionellen Fachkräften und dem Verhältnis zwischen professionellen Akteuren/-innen der Regelversorgung und ihren Patientinnen/ Frauen. Fehlende sprachliche Kompetenzen der Sprachmittlerin im Themengebiet der geburtshilflichen Versorgung, die Konfrontation mit interkulturellen Unterschieden und eine Unsicherheit der professionellen Akteure/-innen im Umgang mit Sprach- und Integrationsmittlung beeinflussen die Tätigkeit. Daneben bieten unterschiedlich praktizierte Rollenverständnisse der SprInterinnen Chancen und Konfliktpunkte in der Zusammenarbeit im Versorgungsnetzwerk Schwangerer und junger Mütter.

Diskussion: Die Bedeutung der Arbeit der Sprach- und Integrationsmittlerinnen für eine bedarfsgerechte geburtshilfliche Versorgung von Frauen mit Sprachbarrieren bietet Chancen für eine öffentliche Diskussion um die Anerkennung des Tätigkeitsprofils. Anpassung und Ausbau von themenspezifischen Qualifikationsmaßnahmen können dabei helfen, Kompetenzen zur Übernahme einer Brücken- und Lotsenfunktion im Sprachund Integrationsmittlungsprozess zu entwickeln und nachfolgend zu praktizieren. Dies impliziert abgesicherte Finanzierungsmöglichkeiten für die Hinzuziehung von Sprach- und Integrationsmittlerinnen in die Regelversorgung von Schwangeren und jungen Müttern und eine Implementierung in interprofessionelle Netzwerkstrukturen.
\end{abstract}

D Open Access. (c) 2021 Angela Rocholl, Ute Lange, published by Sciendo. (cc) Br-NC-ND This work is licensed under the Creative Commons Attribution-NonCommercial-NoDerivatives 4.0 License. 


\section{Keywords}

health care for pregnant migrants - language and integration mediation - role understanding - network function

\section{Keywords}

geburtshilfliche Versorgung - Migrantinnen - geflüchtete Frauen - Sprach- und Integrationsmittlung - Rollenverständnis - Netzwerkfunktion

\section{EINLEITUNG/HINTERGRUND}

Sprachliche Verständigung ist eine Grundvoraussetzung für den Zugang zu Leistungen des Sozial- und Gesundheitswesens und sozialer Teilhabe (Ali \& Watson, 2018; Divi et al., 2007; Eickhorst, 2015; Wolter et al., 2007). Die Migrationsbewegungen in den 90er Jahren und die damit einhergehende Notwendigkeit von Übersetzungen überstieg die Ressourcen herkömmlicher Dolmetscherdienste. So entstand auf lokaler Ebene und in Abgrenzung zum/zur Dolmetscher/-in das Tätigkeitsfeld der Sprach- und Integrationsrmittler/-innen mit dem Schwerpunkt auf fachspezifischem Dolmetschen und soziokulturellem Vermitteln (Becker et al., 2010). Einige politische Stimmen bewerten den Einbezug von Sprach- und Integrationsmittler/-innen als Hemmnis für eine fortschreitende Integration (Leanza, 2005). Die Erkenntnis, dass die Sprach- und Integrationsmittler/innen auch als Vorbilder und Motivation zur Integration dienen können, bleibt häufig im Hintergrund der Diskussion (Hadziabdic \& Hjelm, 2014; Ledyaikina et al., 2007).

Die Sprach- und Integrationsrmittler/-innen werden zumeist zweckgebunden auf kommunaler Ebene finanziert. Die Mitarbeiter/-innen des Sprach- und Integrationsmittlerdienstes SprInt (SprInter/-innen) in Essen begleiten Menschen mit fehlenden oder unzureichenden Deutschkenntnissen unter anderem zu Terminen in kommunalen Behörden, zu professionellen Akteuren/-innen im Sozial- und Gesundheitswesen oder bei Gericht und unterstützen Fachkräfte in der Kommunikation mit den Klienten/-innen (SprInt Servicestelle, 2020).

$\mathrm{Zu}$ den professionellen Akteure/-innen zählen im Kontext der beschriebenen Studie Gynäkologen/innen sowie Hebammen in klinischen und ambulanten Versorgungsbereichen und Fachkräfte aus dem Sozialwesen im Bereich Schwangerschaftsberatung.

ImJahr2018hatteninDeutschland24\%allerNeugeborenen Mütter mit ausländischer Staatsangehörigkeit (Destatis Statistisches Bundesamt, 2019). Kulturell bedingte Unterschiede in den Vorstellungen zu Gesundheit und Krankheit und mangelnde Sprachkenntnisse bedingen häufig Hindernisse im Zugang zur gesundheitlichen Regelversorgung (Borde, 2018; McKnight et al., 2019; Origlia Ikhilor et al., 2019; Razum \& Spallek, 2015). Das Projekt RundUm, finanziert vom Europäischen Asyl-,
Migrations- und Integrationsfond (AMIF), zielte auf eine verbesserte Versorgung von Migrantinnen aus Drittländern mit Sprachbarrieren durch Finanzierungsmöglichkeiten von Sprach- und Integrationsmittlerinnen während der Schwangerschaft, Geburt und der nachgeburtlichen Zeit und dem Auf- und Ausbau eines transkulturellen Netzwerks in Essen ab. Neben der Informationweitergabe an die Akteure/-innen aus dem Sozial- und Gesundheitswesen zu den Möglichkeiten zur Buchung von Sprach- und Integrationsmittlerinnen, stand eine Weiterbildung der SprInt-Mitarbeiterinnen zu den Themenbereichen Schwangerschaft, Geburt, Wochenbett und geburtshilflichen Versorgungsstrukturen und die Durchführung von Interviews mit den Sprach- und Integrationsmittlerinnen im Fokus des Projektes. Beteiligt waren das Bildungsinstitut im Gesundheitswesen (BiG), der Vermittlungsservice für Sprach- und Integrationsmittlung (SprInt) in Essen und der Studienbereich Hebammenwissenschaft an der Hochschule für Gesundheit Bochum (HS Gesundheit). Die Projektzeit lief von Juli 2018 bis Juni 2020.

Der Sprach- und Integrationsmittlerdienst SprInt ist seit 2003 auf lokaler Ebene mit mittlerweile 120 Sprach- und Integrationsmittlern/-innen tätig (Aallali et al. 2019).

Für das Projekt wurden 23 angestellte Sprach- und Integrationsmittlerinnen aus acht Ländern mit $20 \mathrm{zu}$ übersetzenden Sprachen ausgewählt. Aufgrund von ethnischen und religiösen Gründen und zur Erhöhung der Akzeptanz in einem intimen und sensiblen Themenfeld wurden nur weibliche Sprach- und Integrationsmittlerinnen in das Projekt einbezogen.

Bisher veröffentlichte internationale Studien beschäftigten sich neben der Notwendigkeit der Sprachmittlung im medizinischen Bereich (David, 2017; Divi et al., 2007), den Kosten (Brandl et al., 2020) oder den Qualifikationsmöglichkeiten (Wächter \& Vanheiden, 2015) vor allem mit den Rollenstrukturen (Bischoff et al., 2012; Bischoff \& Hudelson, 2010; Leanza, 2005) in der Sprachmittlung. Eine Befragung von Sprach- und Integrationsmittlerinnen im geburtshilflichen Setting fand dabei nur bei Bischoff $(2012 ; 2010)$ statt. Diese waren im Gegensatz zu den Sprach- und Integrationsmittlerinnen von SprInt Essen jedoch nur im klinischen Umfeld tätig. Das Projekt RundUm bot die Möglichkeit, Erkenntnisse zu dem noch wenig beforschten Tätigkeitsfeld aus der Perspektive der Sprach- und Integrationsmittlerinnen zu erfassen. Aus diesem Grund wurden mit ihnen 
Expertinneninterviews geführt. Leitend waren dabei folgende Forschungsfragen:

Welche Herausforderungen sind mit der Tätigkeit als Sprach- und Integrationsmittlerinnen in der Begleitung von Schwangeren/jungen Müttern verbunden?

Wie gestalten sich die Interaktionen im Rahmen der Begleitungen?

Welches Rollenverständnis zeichnet die Sprach- und Integrationsmittlerinnen in ihrer Tätigkeit aus?

Die im Rahmen der Interviews generierten Daten sollten Informationen über die situativen oder institutionellen Kontexte beim Vollzug ihrer Tätigkeit geben und die Vorstellungen und Werte für die Ausübung ihrer Tätigkeit von ihrem eigenem Standpunkt erfassen (Friebertshäuser et al., 2013).

Die Begleittermine gelten dabei gemäß Goffman als „soziale Veranstaltungen“ (2009, p. 34), bei denen Schwangere und junge Mütter, die Sprach- und Integrationsmittler/-innen und die professionellen Akteur/-innen des Gesundheits- und Sozialsystems aufgrund eines zeitlich und räumlich begrenzten Ereignisses aufeinandertreffen. Verschiedene Bezugspersonen messen dabei einer bestimmten Rolle unterschiedliche Bedeutung bei (Merton, 1957 in Abels, 2004). Ziel der Forschung war es, Informationen zu den Inhalten der Interaktionen in der Tätigkeit der Sprach- und Integrationsmittlerinnen und damit verbundene mögliche „Intra- und Interrollenkonflikte“ (Dreitzel, 1980 in Abels, 2004) zu identifizieren und zu analysieren.

\section{METHODE}

\section{Design}

Aufgrund des theoretisch noch wenig erforschten Gegenstandsbereiches wurde ein qualitatives Forschungsdesign in Form von leitfadengestützten Expertinneninterviews gewählt (Gläser \& Laudel, 2010). Die interviewten Sprach- und Integrationsmittlerinnen fungieren dabei als Expertinnen, da sie über ein spezifisches Praxis- oder Erfahrungswissen verfügen. Dieses bezieht sich auf einen klar begrenzten Problemkreis und umfasst die Möglichkeit, das Handlungsfeld sinnhaft zu strukturieren (Bogner et al., 2014). „Die gewonnenen Daten erfassen Deutungen, subjektive Wahrnehmungen, individuelle Relevanzsetzungen und handlungsleitende Orientierungen bezüglich eines Tätigkeitsfeldes“ (Hollstein, 2006). Die Methode hat das Ziel, eine erste Orientierung im Feld, eine Schärfung des wissenschaftlichen Problembewusstseins und schließlich nach Auswertung und Interpretation der Daten eine Thesengenerierung herbeizuführen (Bogner et al., 2014). Der Forschungs- und Analyseprozess wurde regelmäßig nach den Gütekriterien qualitativer Forschung (Mayring,
2016)imRahmenvonInterpretationsgruppen Treffenunter Beteiligung von Hebammenwissenschaftlerinnen und den Verantwortlichen der Projektkooperationspartner/innen diskutiert und reflektiert.

\section{Feldzugang}

Durch die Beteiligung von SprInt als kooperierende Institution wurde der Zugang $\mathrm{zu}$ den Sprach- und Integrationsmittler/-innen organisiert. SprInt bietet seinen Mitarbeiter/-innen regelmäßig Fort- und Weiterbildungsangebote an, die diese als Arbeitszeit verbuchen können. Durch die vorangegangenen Lehreinheiten war die Forscherin den Sprach- und Integrationsmittlerinnen bekannt und die Gespräche wurden in vertrauensvoller Atmosphäre geführt.

\section{Sample}

Für das Projekt wurden Sprach- und Integrationsmittlerinnen ausgewählt, die Erfahrungen mit Begleitungen von Schwangeren und jungen Müttern zu Terminen der gesundheitlichen Versorgung hatten. Von den angesprochenen 25 Mitarbeiterinnen, nahmen 23 regelmäßig an Schulungen zu geburtshilflichen Themen teil. Sie verfügten alle mindestens über ein B2-Deutsch-Sprachniveau. Für die Interviews, deren Teilnahme freiwillig war, konnten 14 Sprach- und Integrationsmittlerinnen gewonnen werden. Dezidiertere Informationen zum Sample können entsprechend der Bewertung der Ethikkommission an der Hochschule für Gesundheit in Bochum aufgrund der Spezifität der personenbezogenen Daten und zur Vermeidung einer möglichen Reidentifizierung nicht gegeben werden.

Tabelle 1: Interviewteilnehmerinnen.

\begin{tabular}{c|c}
\hline $\begin{array}{c}\text { Anzahl der Teilnehmerinnen } \\
\text { zu Sprint }\end{array}$ & 14 \\
\hline $\begin{array}{c}\text { Spannbreite Dauer Zugehörigkeit } \\
\text { Herkunftsländer }\end{array}$ & $\begin{array}{c}\text { Irak, Syrien, Libanon, Marokko, } \\
\text { Tunesien, Afghanistan, Iran, } \\
\text { Kosovo, Albanien, Armenien }\end{array}$ \\
\hline Übersetzte Sprachen & $\begin{array}{c}\text { Kurmandschi, Arabisch in unter- } \\
\text { schiedlichen Dialekten, Serbok- } \\
\text { roatisch, Armenisch, Russisch, } \\
\text { Darsi, Farsi, Paschtu, Französisch, } \\
\text { Englisch }\end{array}$ \\
\hline
\end{tabular}

\section{Durchführung /Datenerhebung}

Über einen Zeitraum von drei Monaten wurden 14 Interviews mit teilstrukturierten Interviewleitfäden geführt. Diese fanden in den Räumlichkeiten der Geschäftsstelle SprInt in Essen statt. Die Teilnehmerinnen konnten die Terminwahl selber gestalten. Die Länge 
der Interviews variierte von 17-50 Minuten. Ein teilstrukturierter Leitfaden diente der thematischen Orientierung, die Reihenfolge der Fragen variierte durch den narrativen Prozess der Interviewten. Innerhalb der Erhebungsphase kam es zu Modifizierungen einzelner Fragen durch den im Forschungsprozess erworbenen Wissenszuwachs der Forscherin (Bogner et al., 2014). Die digital aufgezeichneten Interviews wurden nach lautsprachlichen Regeln inklusive grammatikalischer und semantischer Fehler transkribiert. Sie wurden aus Gründen der Anonymisierung in der Weiterbearbeitung fortlaufend nummeriert. Die Fragen des Interviewleitfadens wurden in vereinfachter Sprache formuliert und auf das Sprachniveau der Befragten angepasst.

Tabelle 2: Interviewleitfaden.

Interviewleitfaden für SprInterinnen zur Begleitung von Schwangeren/jungen Müttern / Familien im Rahmen des Projektes RundUm

1. Welche Sprachen übersetzt du?

2. In welchen Bereichen hast du Frauen und junge Mütter schon einmal begleitet?

3. Wo siehst du die größten Herausforderungen in der Sprachmittlung?

4. Wie empfindest du den Umgang der professionellen Akteure (Ärzte/-innen, Hebammen, Sozialarbeiterinnen) mit Sprachmittlung und mit dir?

5. Wo siehst du Herausforderungen in deiner Rolle als Sprach- und Integra-tionsmittlerin?

6. Was hast du für Erfahrungen gemacht, wie die Akteure/-innen mit der Frau bezogen auf die Sprachbarriere umgehen?

7. In welchen Situationen ziehst du kulturelle Eigenarten der Schwangeren/jungen Mutter in die Sprachmittlung ein?

8. Was glaubst du, gelingt dir in der Sprachmittlung besonders gut, was nicht so gut?

9. Was würdest du dir bei den Begleitungen manchmal wünschen? 10. Was wünschst du dir für dich selber?

\section{Datenanalyse}

Die Datenauswertung leitete sich vom Format des Expertinneninterviews abunderfolgte nach einerqualitativ interpretativen Auswertungsform der Sozialforschung nach Meuser und Nagel (2013). Die Transkripte wurden mithilfe von MAXQDA offen codiert. Es wurden nach thematischer Relevanz Themen herausgesucht, die für die Ausgangsfragestellungen wichtig sind. Im Schritt des thematischen Vergleichs wurden Unterkategorien und Hauptkategorien herausgearbeitet (siehe Abb. 1). Dabei wurden gleiche Themenbereiche aus allen Interviews gebündelt und die einzelnen Einteilungen im Rahmen von Interpretationsgruppen nochmals diskutiert und reflektiert. Die zugehörigen Interviewinhalte wurden tabellarisch dargestellt, um eine Vergleichbarkeit zu ermöglichen. Durch eine „komparative Analyse“ fielen Passagen mit besonderer interaktiver und metaphorischer Dichte auf (Bohnsack, 2014). Der thematische
Vergleich wurde neben der tabellarischen Darstellung noch in deskriptiver Formulierung ausgearbeitet. Die ausgewählten thematisch relevanten Aussagen wurden im nächsten Schritt einer detaillierten, formulierenden Interpretation unterzogen (Bohnsack, 2014).

\section{Ethische Aspekte}

Im Rahmen einer Informationsveranstaltung wurde den SprInt-Mitarbeiterinnen die Durchführung der Interviews vorgestellt. Die Freiwilligkeit der Teilnahme und die Abgrenzung der Forscherin zur Institution des Arbeitgebers wurden hervorgehoben, insbesondere da die Zeit der Interviewteilnahme als Arbeitszeit angerechnet wurde. Die SprInterinnen wurden über ihr Einverständnis zum Interview, der digitalen Aufnahme und den Umgang mit den gewonnenen Daten aufgeklärt. Für das Vorhaben wurde ein Ethikantrag an der Hochschule für Gesundheit Bochum eingereicht, der ein positives Votum erhielt.

\section{ERGEBNISSE}

Im Auswertungsprozess konnten fünf Hauptkategorien identifiziert werden: Beeinflussende Faktoren zur Ausübung der Tätigkeit, Umgang mit interkulturellen Aspekten, Interaktionen zwischen den an den Begleitungen Beteiligten, Bedeutung der Sprachmittlung für die gesundheitliche Versorgung der Klientinnen und Bedeutung der individuellen Ausgestaltung der Tätigkeit als Sprach- und Integrationsmittlerin.

\section{Beeinflussende Faktoren zur Ausübung der Tätigkeit}

Die Faktoren, die die Rahmenbedingungen zur Ausübung der Tätigkeit der Sprach- und Integrationsmittlerinnen betreffen, basieren laut der Interviewdaten auf rechtlichen, organisationellen und fachlichen Grundlagen. Die Aussagen der Befragten veranschaulichen, dass die rechtlichen Vorgaben bezüglich des Datenschutzes den Zugang zu ihren Klientinnen erschweren. Eine Befragte kritisiert, dass aufgrund fehlender Weiterleitung des Namens oder der Telefonnummer eine vorherige Kontaktaufnahme zur Generierung von Vorinformationen unmöglich ist. Dies erschwert bei dem Termin eine Identifizierung der Klientin, sodass diese unter Umständen erst durch Informationen der Institution oder die Kontaktaufnahme mit dem Sprachmittlerservice geschieht:

„Wir sagen, ja zum Beispiel, zehn Uhr Termin: Wie heißt die Patientin? - Wissen wir nicht. Geburtsdatum - wissen wir nicht. Ja dann warten wir. Telefon ist die nicht erreichbar zum Beispiel, ne. Dann stehen wir 
Ähm, und dann hab ich das auch öfter immer gesagt: „Sie können auch den/es gibt Flüchtlingsberatung hier in der Nähe.".. Ich kenn mittlerweile fast in jedem Stadtteil, wo eine ist und dann teil ich das auch mit. Und die (die Kinderärzte) empfinden das halt als große Hilfe...... Interview 11 (Zeile 81ff)

„Diakonie oder Caritas oder Plan B. Und ich sag: Geh zu dieser Sozialarbeiterin, können beraten, sie können euch helfen." Interview6 (Zeilen 92ff)

Er fragt ja: „Hat sie noch Fragen?", und dann kläre ich sie darüber auf oft, was sie noch fragen sollte vielleicht.....Müssen wir ein bisschen nachhelfen manchmal, ja. Interview 8 (Zeile 49ff)

"Haben Sie das gefragt?" oder „Wissen Sie diese Situation?" oder "Soll ich Ärztin fragen?", dann sagt sie „Ja bitte". Ja wenn ich fühle, dass das ist nicht genug Information, die Damen hatte schon angefragt, ich versuche sie helfen, dass sie mehr Fragen stellen. Interview1 (Zeilen165ff)

Abbildung 1: Codierbeispiel.

zehn Minuten, fünfzehn Minuten. Und die Klientin sitzt schon da!“ Interview 7 (Zeilen $115 \mathrm{ff}$ )

Vorinformationen werden von den SprIntMitarbeiterinnen durchweg als sinnvoll und hilfreich bewertet.

„Dann frag ich mal halt wie viele Kinder, allgemeine Information, ob sie Erkrankung hat und so, das hilft alles bei der Ausfüllen der Anmeldebogen." Interview 9 (Zeilen 41ff)

Als Möglichkeit zum Erhalt von Informationen geben die Sprach- und Integrationsmittlerinnen zum einen Telefonkontakte an, deren Zeitaufwand jedoch nur bei ausdrücklicher Anordnung bezahlt wird, zum anderen Informationen durch die Klientin im direkten Kontakt oder durch die professionellen Akteure/-innnen aus dem Sozial- und Gesundheitswesen. Als Zugangsbarrieren vonseiten der Klientinnen werden Desinteresse oder technische Unkenntnis zur Bedienung von Mobiltelefonen beschrieben.
„Und ich habe ihr gesagt, dann soll sie (die Frau) mir das kopieren und zuschicken lassen, damit ich das ganze Termine eintragen bei mir und sie sagte, sie kann mich nicht anrufen. Dann hab ich geguckt. Wieso? Ja sie kann nicht, also sie hat keine Ahnung wie man Handy benutzt [...] oder sie soll mich anrufen, dann kläre ich das. Und bis heute hat sie sich nicht mehr gemeldet. "Interview 4 (Zeilen 25ff)

Nebendenfachlichenund sprachlichenHerausforderungen zeigen sich Aspekte zur professionellen Abgrenzung, einem variierenden Verständnis zur Rolle von Sprachund Integrationsmittlerinnen von Seiten der Klientinnen und der Einfluss der Anwesenheit von Begleitpersonen im Rahmen des interprofessionellen Kontakts.

Bezüglich ihrer eigenen fachlichen Kompetenzen geben die SprInt-Mitarbeiterinnen an, dass durch die deutsche Grammatik der Wechsel von einer in die andere Sprache erschwert sei und die Qualität der Sprachmittlung auch von ihrer jeweiligen Tagesform abhänge. Im Vergleich zu anderen Einsatzgebieten, bei denen das Niveau der Übersetzung teilweise nicht sehr anspruchsvoll sei, ist speziell im medizinischen Bereich eine vollständige 
Kenntnis der Fachbegriffe wichtig. Diese sei aber nur realisierbar, wenn die entsprechenden sprachlichen Kompetenzen inklusive der Vermittlung von Fachtermini ermöglicht wurden.

„Oder zum Beispiel bei der Diakonie [...] dann hat man zum Beispiel im Gespräch schon gehört, dass sie ne Krankheit hat, die sie dann auf Arabisch genannt hat, ähm, die ich zwar übersetzt habe, aber nicht weiß, was das wirklich heißt. Und ich habe mich dann zuhause informiert, für den nächsten Termin, [...] damit das halt, ja, professioneller ist" Interview 11 (Zeilen 33ff)

Insbesondere die Übersetzung aus dem Arabischen oder ins Arabische sei eine Herausforderung, da diese Sprache viele Dialekte mit unterschiedlichen Begriffsbedeutungen enthält. Die Sicherstellung des Verständnisses ist daher selbst bei Muttersprachlerinnen nicht einfach und bedarf in vielen Kontexten einer erhöhten Konzentration.

In Situationen, in denen die Klientin über Deutschkenntnisse verfügt, braucht die Sprach- und Integrationsmittlerin nur im Bedarfsfall zu unterstützen. Sie versucht vermittelnd zu übersetzen und wägt ab, wo Informationen zum Sprachmittlungsvorgang notwendig oder verzichtbar sind.

Sprach- und Integrationsmittlerinnen erhalten Einblicke in Bereiche, die für Außenstehende normalerweise nicht zugänglich sind, und müssen sich spontan auf neue Situationen einstellen. Das ihnen von der Klientin entgegengebrachte Vertrauen empfinden mehrere Interviewte in schwierigen Situationen als belastend und überfordernd.

„Also die Frau hat Vertrauen zu mir. [...] Sie hat auch zu mir gesagt, bevor sie reinkam: "Ich bin stark, weil du jetzt bei mir bist!" [...] Also als ich gemerkt, die ist bewusstlos, [...] ich hab gemerkt, dass sie nicht mehr atmet [...]. Da habe ich Angst gehabt.“ Interview 4 (Zeilen 59ff)

Die Interviewten geben den Wunsch an, sich nicht zu nah von belastenden Krankheitsbildern konfrontieren zu lassen und dass das Erleben dramatischer Situationen zu einer emotionalen Belastung und innerer Beteiligung führt. Dies wird durch das Fehlen von Ansprechpartnern/innen auf institutioneller Ebene verstärkt.

„Und allein nachts, wo ich dastehe, ich kann keine im Büro erreichen, ich kann mit keiner reden, wo ich auch vielleicht Hilfe holen. Was soll ich machen? Was soll ich tun? Und so. Das war schwierig." Interview 4 (Zeilen 59ff)
Einige Interviepartnerinnen schildern ihre Versuche, sich abzugrenzen, indem sie die Weitergabe ihrer Telefonnummer an die Klientinnen zur weiteren Kontaktaufnahme ablehnen. Dies führt hin zu der Aussage einer Sprach- und Integrationsmittlerin:

„Und ich versuche so, dass selbe Straßenbahn, dass ich in eine andere schon einsteige [...] Ich will nicht die ganze Stunde lange in Straßenbahn mit die Frage attackiert werden." Interview 5 (Zeilen 71ff)

\section{Umgang mit interkulturellen Aspekten}

Im Rahmen ihrer Tätigkeit als Sprach- und Integrationsmittlerin ist die Vermittlung interkultureller Aspekte für die Interviewten sehr relevant und beinhaltet auch die Erklärung der Unterschiede zwischen dem deutschen Gesundheitssystem und dem des Heimatlandes. Verbal vermittelte Informationen sind oftmals notwendig, da durch einen großen Anteil an Frauen mit Analphabetismus Printmedien nur begrenzt eingesetzt werden können. Die Befragten geben je nach Thema an, die Klientinnen darüber aufzuklären, dass aus den Heimatländern gewohnte Verhaltensweisen in Deutschland nicht üblich sind.

„Aber für dich so ist besser, wenn die Ärztin sagt was, du musst das akzeptieren und machen. Bei uns die Problem ist, viele kurdische Frauen die haben [...] ja Hausgeburt allein gemacht. Hier ist das nicht so und die wollen so leicht das machen hier auch, aber es geht nicht. Die müssen diesen verstehen." Interview 6 (Zeilen 111ff)

Dabei sehen es die Teilnehmerinnen während des Übersetzungsprozesses kritisch, dass die Klientinnen zuweilen Schwierigkeiten haben, Empfehlungen und vorgeschlagene Handlungsabläufe zu akzeptieren. Dies kann selbst in lebensbedrohlichen Situationen geschehen.

„Die Hebamme ähm, irgendwie: Sie sterben da!, die gesagt: Ja dann Gott wollte, dass ich sterbe. Also, das war für sie egal, Hauptsache mehr Kinder. "Interview 7 (Zeilen 112ff)

Im Kontakt mit den professionellen Akteuren/-innen im Gesundheits- und Sozialwesen versuchen die Sprach- und Integrationsmittlerinnen, mit Erklärungen Vorurteile zu entkräften. Auch wenn nach Angaben der Interviewten einige der professionellen Akteure/-innen über kulturelle Eigenarten und Gewohnheiten von Frauen aus Drittstaaten informiert sind, empfinden viele deren Umgang mit den betreuten Frauen als wenig kultursensibel. Zusätzlich stellen sie fest, dass unbekannte Methoden der Diagnostik 
und die Anwesenheit von männlichen Akteuren bei den Klientinnen zu Angst und Ablehnung führen.

Nach Aussage der SprInterinnen hängt der Grad der Notwendigkeit der Unterstützung vom Alter der Klientinnen und der Länge ihres Aufenthalts in Deutschland ab.

\section{Interaktionen zwischen den an den Begleitungen Beteiligten}

Aus der Perspektive als Mittlerin im Prozess der Kommunikation zwischen Akteuren/-innen und Klientinnen formulierten die Sprach- und Integrationsmittlerinnen prozesshinderliche und prozessfördernde Verhaltensweisen in der Interaktion. Als Bindeglied zwischen Akteur/-in und Klientin berichten die Frauen, mit möglicher Unzufriedenheit und hohen Ansprüchen der Klientinnen konfrontiert zu werden. Dies wird teils als Undankbarkeit der Klientinnen gegenüber den angebotenen Unterstützungsmaßnahmen wahrgenommen. Die Sprach-und Integrationsmittlerinnen merken an, dass die Klientinnen häufig die Relevanz der detaillierten Informationen nicht erkennen. Verstärkt wird dies durch den Einfluss von Begleitpersonen, wie z. B. Partner und Familienmitglieder, die versuchen, die Gesprächsinhalte und Gesprächsführung zu beeinflussen. Die Anwesenheit und Dominanz von anwesenden Partnern stellen dabei die größten Herausforderungen dar.

„Die versuchen sich einzumischen, die lassen nicht den Klienten selber alles ausreden, wenn die Frage gestellt. Und manchmal ist die Situation, der Sachbearbeiter bitten, dass der Begleiter rauszugehen." Interview 5 (Zeilen 47ff)

Auf der anderen Seite vermitteln viele professionelle Akteure/-innen des Gesundheits- und Sozialwesens, dass sie den Übersetzungen misstrauen und den Sprach- und Integrationsmittlerinnen nicht zutrauen, den Sinn des Gesagten in größeren Zusammenhängen zu verstehen und weiterzugeben.

„Aber ich habe das auch schon ganz oft erlebt, dass die Akteure gesagt haben, ne, nach kurzen Abschnitten gesagt haben: „So, übersetzen sie das bitte jetzt", so, darauf richtig bestanden haben, dass ich das Stück für Stück. "Interview 2 (Zeilen 39ff)

Sie stellen fest, dass Klientinnen häufig die Termine nicht einhalten oder auf die Hinzuziehung von Übersetzern/innen aus dem eigenen Bekanntenkreis bestehen, zudem stellen einige von ihnen hohe Anforderungen und sind trotz der Vielzahl an Angeboten nicht zufrieden.
„Generell die sind nicht zufrieden. [...] Ja, das ist das Problem. Das tut mir weh! Weil ich gucke und ich sehe von welchem Angebot [...]. Die kriegen überall Hilfe. Wirklich Tip-Top, von A-Z. Und wenn ich vergleiche in unserer Zeit, trotzdem wir waren zufrieden mehr als diejenigen jetzt. “Interview 1 (Zeilen 117ff)

Diese Haltung der begleiteten Frauen beeinflusst im Gegenzug das Verhalten der professionellen Fachkräfte im Gesundheits- und Sozialwesen. Die Interviewten geben an, dass neben vielen Akteuren/-innen, die sich auf die kulturellen Eigenarten der Frauen und dem damit verbundenen zeitlichen Mehraufwand einstellen, andere mit wenig Geduld und fehlender Wertschätzung den Klientinnen gegenüber reagieren.

„Ich hatt zum Beispiel auch einen Fall gehabt ähm, die Frau hatte drei Kinder zu Hause entbunden, in Albanien, immer mit Frauen. Und sie kannte nicht, also gynäkologischen Stuhl, [...] und das war natürlich ein Frauenarzt. Und ähm, ja wir haben dann versucht, oder, zu erklären, dass sie jetzt da drauf muss und dann, dann wollte sie nicht! Die war total, sie hat angefangen zu weinen und weil sie hat gesagt: 'Ich hab die Kinder zu hause entbunden. Da ist ein Mann vor mir' Und so Und da war der Gynäkologe sehr unfreundlich, weil er meinte, er hätte jetzt keine Zeit mehr hier zu verlieren." Interview 10 (Zeilen 83ff)

Die Sprach- und Integrationsmittlerinnen merken an, dass die professionellen Fachkräfte einen detaillierten Erklärungsbedarf teils nicht erkennen und häufig nur ein eingeschränktes Interesse gegenüber den Klientinnen zeigen. Kompromissbereitschaft in der Behandlung zeigen viele Akteure/-innen erst nach Aufklärung durch die SprInt-Mitarbeiterinnen.

Mehrheitlich erhalten die Sprach- und Integrationsmittlerinnen eine positive Resonanz zu ihrer Tätigkeit von den professionellen Akteuren/-innen. Die SprInt-Mitarbeiterinnen betonen die Bereitschaft der professionellen Akteure/-innen, sie bei auftretenden Wissenslücken erklärend $\mathrm{zu}$ unterstützen. Jedoch werden auch Situationen beschrieben, in denen diese versuchen,die persönliche Meinung der Sprach- und Integrationsmittlerin zu erfragen und sie damit zwingen, die ihr zugeschriebene Rolle $\mathrm{zu}$ ändern und in den Aushandlungsprozess mit der Klientin einzugreifen.

„Was meinen Sie darüber? Ist das so oder so? [...] Ich sagte: Ich kann leider nicht Ihnen helfen. Was sie gesagt hat, hab ich das übersetzt. Und was ich, meine Meinung zählt das nicht." Interview 5 (Zeilen 79ff) 
Die Frauen schildern auch ein Unverständnis von Seiten der professionellen Akteure/-innen, warum das Übersetzen in eine andere Sprache sprachlich teils umfangreicher ausfällt, als es der deutschen Urfassung entspricht.

„Und manchmal müssen wir sagen, das selbe Wort haben wir das nicht, wir müssen also so rum, herum beschreiben." Interview 5 (Zeilen 87ff)

Auch wenn die Sprachmittlung nach Meinung der Interviewten grundsätzlich positiv empfunden wird, berichten sie von Zwischenfällen, wie der Hinderung am Einlass in einen Kreißsaal entgegen des Wunsches der Klientin.

„Da haben die mich gar nicht reingelassen. Ich bin wirklich umsonst dortgeblieben. Der Mann war bei mir, er hat gesagt: Ne, ich schaff nichts, ich kann nicht. Die Frau war alleine. “Interview 4 (Zeilen 52ff)

ZudembemerkendieSprach-undIntegrationsmittlerinnen, dass die professionellen Fachkräfte das zeitabhängige Buchungssystem ignorieren, was dazu führt, dass Termine mit langen Wartezeiten dann letztlich nicht mit der Sprach- und Integrationsmittlerin durchgeführt werden können.

\section{Bedeutung der Sprachmittlung für die gesundheitliche Versorgung der Klientinnen}

Eine Sprach- und Integrationsmittlerin berichtet, dass einige professionelle Akteure/-innen Frauen ohne die Begleitung einer Sprach- und Integrationsmittlerin nicht behandeln. Durch die Anwesenheit letzterer empfinden die Klientinnen ein Gefühl der Sicherheit, des Vertrauens und Schutz. Dies wird durch gleiche Herkunft noch verstärkt.

„Kannst du Deutsch? Kannst du Kurdisch? Auch du bist von meine Stadt oder von die Nähe von meine Stadt, dann sag dir mein Problem und du kannst mir helfen!“ Interview 6 (Zeilen 52ff)

Gerade im geburtshilflichen Kontext bevorzugen die Klientinnen weibliche Sprach- und Integrationsmittlerinnen. Nach Meinung der SprIntMitarbeiterinnen erleichtert ihre Arbeit die Versorgung der Frauen und führt im Normalfall zu Problemlösungen. Auch eine korrekte Medikamenteneinnahme kann durch Sprachmittlung gewährleistet werden.

„Die war in fünfte Monat. Und die hat gesagt, zwei Monaten, ich habe Medikamente falsch genommen. Ich hatte kein Dolmetscher." Interview 6 (Zeilen 115ff)
Selbstkritisch wird angemerkt, dass Sprach- und Integrationsmittlerinnen die Klientinnen in ihren Fragen an die Fachkräfte beeinflussen können. Die Interviewten betonen, dass ihre Tätigkeit zu Meinungsänderungen bei den Klientinnen führen kann und dass sie teils Ratschläge für ein weiteres Vorgehen vermitteln, womit sie ihre reine Übersetzerinnenrolle verlassen.

„Wir wissen, welche Möglichkeiten hat die Dame. Weitere Untersuchungen und sowas, sagen wir das. “ Interview 1 (Zeilen 86ff)

Eine konstante Begleitung der Frauen wird als anzustrebendes oberstes Ziel bewertet. Jedoch äußern einige Sprach- und Integrationsmittlerinnen das Phänomen, dass ihre Anwesenheit den Frauen die Verantwortung $\mathrm{zu}$ nehmen scheint, eigene sprachliche Kompetenzen erwerben und einsetzen zu müssen. Auch die Möglichkeit einer falschen Übersetzung mit den daraus resultierenden Folgen schließen die Sprach- und Integrationsmittlerinnen bei anderen Kolleginnen, jedoch nicht für ihre eigene Arbeit, nicht aus.

\section{Bedeutung derindividuellen Ausgestaltung der Tätigkeit als Sprach- und Integrationsmittlerin}

Innerhalb der Interviews wird deutlich, dass die Sprachund Integrationsmittlerinnen ihre Befugnisse und Kompetenzen im Rahmen ihrer Tätigkeit sehr individuell gestalten. Dies wird in ihrer Positionierung innerhalb der kommunikativen Situationen offensichtlich. Der Großteil der interviewten Frauen gibt an, dass sie den Klientinnen durch ihr Fach-und Erfahrungswissen Frageoptionen an die Akteure/-innen vorgeben und die Klientinnen ermutigen, Fragen zu stellen.

„Also wenn, wie gesagt, wenn ich weiß, dass da etwas halt ist, was sie unbedingt mitteilen sollte, oder worauf sie achten sollte, dann sag ich das natürlich, wenn das jetzt wichtig ist...." Interview 11 (Zeilen 93ff)

Auch gibt die Sprach- und Integrationsmittlerin schon im Vorfeld Informationen an die Klientin weiter und beeinflusst damit die nachfolgenden Fragen bei den professionellen Akteuren/-innen. Einige Sprach-und Integrationsmittlerinnen weisen die Fachkräfte auf das Selbstbestimmungsrecht der Schwangeren hin oder schlagen Therapie- und Diagnostiken vor.

„Ich hab die Ärztin gefragt, ob die Damen braucht eine Frucht(wasser)untersuchung oder nicht, weil sie zwei Kinder hat, die nicht in Ordnung sind." Interview 1 (Zeilen 173ff) 
Nur wenige Sprach- und Integrationsmittlerinnen geben $\mathrm{zu}$, jegliche Einflussnahme auf die Gesprächsführung abzulehnen.

Durch ihre Position als verstehende und begleitende Mittlerin hat die Tätigkeit der Sprach- und Integrationsmittlerin auch Effekte aufdieZusammenarbeit mit den professionellen Akteuren/-innen, die an der Versorgung Schwangerer und junger Mütter beteiligt sind. Die Interviewten merken an, durch ihr Netzwerkwissen, die Akteure/-innen in ihrer Arbeit durch weiterführende Informationen $\mathrm{zu}$ unterstützen und Anfragen von Klientinnen an die Akteure/-innen weiterzuleiten, oder selber an Akteure/-innen zu vermitteln.

"[...] hab ich Anruf gekriegt, wo eine Frau, die ist in der Schwangerschaft, sechster Monat, ist erblindet, und die konnte noch ein bisschen Schatten sehen. Gestern rief mich der Mann an [...] und sagte, die Frau sieht jetzt komplett Schwarz mit beiden Augen. Sie hat ein Baby ein Jahr alt. Und da hab ich gesagt:, Ich rufe Sozialarbeiterin an!" Und sie hat sich gekümmert für heute Termin beim Arzt." Interview 4 (Zeilen 83ff)

Gemeinsame Gespräche zwischen Fachkräften und Sprach- und Integrationsmittlerinnen ermöglichen die Durchführung von notwendigen Maßnahmen und machen eine Versorgung effektiver. Basierend auf der langjährigen Übersetzungstätigkeit im medizinischen oder sozialen Bereich verfügt die Sprach- und Integrationsmittlerin über ein weitreichendes fachliches Wissen und gibt dies an die professionellen Akteuren/innen weiter.

„Ich kann manchmal die Sozialarbeiterin zeigen, worum es geht, was müssen die machen, welchen Weg müssen die nehmen. Weil manche davon sind sehr junge Sozialarbeiterinnen, die gerade von der Uni rausgekommen sind. Und wir haben Berufserfahrung. Wir können ihnen zeigen, zum Beispiel: Da ist das und wir können dahin begleiten. Und das ist auch eine erleichterte Arbeit für die Sozialarbeiter." Interview 5 (Zeilen 127ff)

\section{DISKUSSION}

In den 14 Interviews mit den Sprach- und Integrationsmittlerinnen wurden neben vielen individuellen Erfahrungen und Fallbeispielen aus dem Arbeitsalltag auch Gemeinsamkeiten in der beruflichen Ausübung deutlich. Trotz einheitlicher Sprachvoraussetzungen für die Tätigkeitsausübung konnten in einem Einzelfall Textstellen nicht in die Analyse einbezogen werden, da ein Textverständnis beim
Durchlesen nicht erreicht werden konnte. Dass die Daten ausschließlich im Sprach- und Integrationsdienst SprInt Essen erhoben wurden, ist eine Limitation der Studie. Jedoch können die Ergebnisse eine wissenschaftliche Grundlage für weitere Forschung bilden. Sie liefern einen vielseitigen Einblick in das bis dahin wissenschaftlich noch wenig beachtete Tätigkeitsfeld der Sprach- und Integrationsmittlerinnen in der geburtshilflichen Begleitung.

Neben Herausforderungen im Zugang zu Klientinnen und ihren eigenen fachlichen Kompetenzen wird die Bedeutung der kulturellen Mittlerinnenposition über die Übersetzungstätigkeit hinaus hervorgehoben.

Eine sehr heterogene Auslegung ihrer Tätigkeitsinhalte deckt ein diverses Rollenverständnis vonseiten der Sprach- und Integrationsmittleinnen auf. Nicht selten geht dieses über die internen Qualifikationsrichtlinien der gemeinsamen Qualifizierungsmaßen des SprInt Netzwerkes hinaus (SprInt Servicestelle, 2020). Vom reinen „Word-to-WordÜbersetzer“ über die Funktion des „Klienten-Anwalts“ bis zum „Co-Therapeuten“(Bischoff et al., 2012) reicht die Spannbreite der in den Interviews dargelegten gelebten Funktionen. Nicht zuletzt ist dies bedingt durch die geteilten kulturellen Gemeinsamkeiten der Sprach- und Integrationsmittlerinnen und deren vergleichbaren Historien durch eigene Flucht- und Migrationserfahrungen. Diese Faktoren erschweren bei einigen Interviewten eine professionelle Abgrenzung. Sie erklären auch die dargestellten Konflikte im Umgang mit emotionalen Ereignissen und erschweren den oftmals nicht geklärten Beziehungsstatus gegenüber der Klientin. Nicht zuletzt kann diese Übernahme erweiterter Rollenfunktionen vonseiten der professionellen Fachkräfte auch als fehlende professionelle Neutralität und Distanz gewertet werden (Borde, 2007).

Das uneinheitliche Handeln der Sprach- und Integrationsmittlerinnen birgt auch für die begleiteten Schwangeren und jungen Mütter Unsicherheiten im Beziehungsaufbau, da die kontinuierliche Begleitung durch ein und dieselbe SprInt-Mitarbeiterin nicht gewährleistet werden kann und es folglich zur Kollision von Erwartungen kommt.

Auch vonseiten der professionellen Akteure/-innen aus dem Gesundheits- und Sozialwesen wird ersichtlich, dass die Vorstellungen und der Umgang mit Sprach- und Integrationsmittlerinnen sehr variieren und zum Teil auf systemischer Unwissenheit, aber auch mangelndem Kooperationswillen gründen (Hadziabdic \& Hjelm, 2014). Beide Aspekte gehen letztlich auf Kosten der Versorgung der betreuten Frauen. Die Ergebnisse zeigen, dass die Ablehnung einer Behandlung ohne Sprachund Integrationsmittlerin sowie die Durchführung einer Behandlung ohne verfügbare Verständnismöglichkeiten die Qualität der Versorgung relativiert und damit auch 
den Ansprüchen der Agierenden nicht genügen kann. Die Hinzuziehung von Sprach- und Integrationsmittlerinnen ermöglicht den schwangeren Frauen und jungen Müttern, die allseits geforderte Umsetzung der rechtlichen Voraussetzung eines ,decision making“ und eines „no decision about me, without me“ (McKnight et al., 2019). Fehlende Eigeninitiativen und fehlende systemische Voraussetzungen vonseiten der professionellen Akteure, Sprach- und Integrationsmittlung als Teil der täglichen Versorgung Schwangerer mit Sprachbarrieren zu implementieren, werden in den Ergebnissen ersichtlich.

Auf der anderen Seite profitieren die Fachkräfte aufgrund der Mittlerinnenfunktion der Sprach- und Integrationsmittlerinnen vom fundierten Wissen und nutzen dies dazu, Netzwerklücken in den Versorgungssystemen zu schließen. Auch wenn viele der SprInterinnen diese Funktion auf Eigeninitiative und durch ihre langjährigen Erfahrungen ausfüllen, würden sie dafür eine weitreichendere Qualifikation und eine rechtlich gesicherte Verankerung in den Versorgungsstrukturen benötigen.

Die Auseinandersetzung mit Grenzerfahrungen, die die Begleitung in der perinatalen Zeit mit sich bringt, stellt täglich eine große Herausforderung für die Sprach- und Integrationsmittlerinnen dar. Uneinheitliche Qualifikationsinhalte und sehr weit aufgestellte Schulungsinhalte reichen für die Begleitung und die Vorbereitung auf mögliche Situationen in der Schwangerenbetreuung, im Kreißsaal und Wochenbett nicht aus. Für eine effektive und zufriedenstellende Tätigkeit in der geburtshilflichen Begleitung bedarf es der fokussierten Ausbildung und einer weitreichenderen Integration von Sprach- und Integrationsmittlung in die Versorgungsstrukturen in Ergänzung der Tätigkeiten der professionellen Fachkräfte wie Hebammen, Ärzte/innen oder Sozialarbeiter/-innen und damit eines klaren Kompetenzprofils.

Das beschriebene tiefe Vertrauen der Schwangeren und jungen Mütter in die Arbeit der Sprach- und Integrationsmittlerinnen und die dargestellten positiven Effekte der Begleitungen auf die geburtshilfliche Versorgung unterstreichen die bekannten wissenschaftlichen Erkenntnisse zum Nutzen der Hinzuziehung von Sprach- und Integrationsmittlerinnen (Borde, 2018; Brenne et al., 2013; Divi et al., 2007; RundUm. Ergebnisbroschüre, 2020). Der in vielen Fällen beschriebene Idealismus vonseiten der Sprachund Integrationsmittlerinnen über ihre vonseiten der Institution festgelegten Rolle hinaus und ihr zum Teil fundiertes Erfahrungs- und Fachwissen in unterschiedlichen Bereichen beinhaltet Möglichkeiten und Chancen, diese Fähigkeiten für die Integration von Schwangeren und jungen Müttern mit Migrations- oder Fluchthintergrund gewinnbringend einzusetzen.
Der momentane Rückgang der Migrationszahlen darf nicht zu stagnierenden Strukturen oder gar zum Abbau derselben führen, sondern eröffnet Chancen, die Qualifikation von Sprach- und Integrationsmittlerinnen im geburtshilflichen Kontext weiterzuentwickeln und $\mathrm{zu}$ vereinheitlichen. Der dargestellte Bedarf rechtfertigt es, weitere anerkannte Tätigkeitsfelder zu schaffen, die die Netzwerkpotenziale der Sprach- und Integrationsmittlerinnen integrieren. Denkbar dabei wäre eine Kombination zwischen Sozialmittlerin und Sprachund Integrationsmittlerin.

Durch eine staatliche Anerkennung der Tätigkeit mit damit verbundenen einheitlichen Qualifikationsrichtlinien und der Implementierung in die sozialen und gesundheitlichen VersorgungssystememussSprach-undIntegrationsmittlung fester Bestandteil der Versorgungsstrukturen werden. Interprofessionelle Trainings mit den professionellen Akteuren/innen des Gesundheits- und Sozialwesens dienen dazu, voneinander zu lernen und sind wertvoll für die Schaffung von gegenseitigem Verständnis und Respekt (Gün, 2018; Sturman et al., 2018).

Relevante Ergebnisse, die durch zeitlich begrenzt geförderte Projekte wie RundUm generiert wurden, müssen als wissenschaftliche Fundierung Einfluss auf Anschlussfinanzierungen finden. Hier bedarf es noch weiterer wissenschaftlicher Ergebnisse, die sich auf die Perspektiven der professionellen Akteure/-innen aus dem Gesundheits- und Sozialsystem und begleiteten Schwangeren/jungen Mütter gründen. Finanzielle Sicherheiten für die Institutionen des Gesundheits- und Sozialwesens können zu Festanstellungen von Sprach- und Integrationsmittlerinnen in den Institutionen selber und zu einem leichteren Zugang zu den Sprachmittlerdiensten führen und den Schwangeren und jungen Müttern mit ihren Familien dabei helfen, ihre Wurzeln im neuen Heimatland langsam und beständig zu entwickeln.

\section{Dank}

Die Erhebung wurde im Rahmen des Projektes RundUm durch den Asyl, Migration, Integrationsfond (AMIF) finanziert. Wir bedanken uns bei den Sprach-und Integrationsmittlerinnen für die motivierte Teilnahme und den Kooperationspartnern/-innen für die gemeinsame Durchführung des Projektes.

\section{Ethische Prüfung}

Das ethische Clearing wurde von der Ethikkommission der Hochschule für Gesundheit Bochum erteilt.

\section{Interessenkonflikte}

Es bestehen keine Interessenkonflikte 
Aallali, H; Albash, L; Elias, 1; Tadic-Ruzic, R; Schweitzer, H (2019).

„Ohne Sie könnten wir mit unseren Klienten nicht kommunizieren“. In. Sozial Extra, 43 (2), 96-102.

Abels, H. (2004). Einführung in die Soziologie (2., überarb. und erw. Aufl.). Hagener Studientexte zur Soziologie: [8]. Wiesbaden: Springer VS.

Ali, P. A., \& Watson, R. (2018). Language barriers and their impact on provision of care to patients with limited English proficiency: Nurses' perspectives. Journal of Clinical Nursing, 27(5-6), e1152-e1160. https://doi.org/10.1111/jocn.14204.

Becker, C., Grebe, T., \& Leopold, E. (2010). Sprach- und Integrationsmittler/-in als neuer Beruf: Eine qualitative Studie zu Beschäftigungspotenzialen, Angebotsstrukturen und Kundenpräferenzen. Diakonie Wuppertal Im Auftrag des Bundesministeriums für Arbeit und Soziales. https://www.google. $\mathrm{com} /$ search?client=firefox-b-d\&q=Sprah-+und+Integrationsmittl er+als+neuer+beruf.

Bischoff, A., \& Hudelson, P. (2010). Communicating with foreign language-speaking patients: Is access to professional interpreters enough? Journal of Travel Medicine, 17(1), 15-20. https://doi. org/10.1111/j.1708-8305.2009.00314.x.

Bischoff, A., Kurth, E., \& Henley, A. (2012). Staying in the middle: A qualitative study of health care interpreters' perceptions of their work. Interpreting, 14(1), 1-22. https://doi.org/10.1075/ intp.14.1.01bis.

Bogner, A., Littig, B., \& Menz, W. (2014). Interviews mit Experten: Eine praxisorientierte Einführung. Qualitative Sozialforschung. Wiesbaden: Springer VS.

Bohnsack, R. (2014). Rekonstruktive Sozialforschung: Einführung in qualitative Methoden (9., überarb. und erw. Aufl.). UTB: 8242 : Erziehungswissenschaft, Sozialwissenschaft. Budrich.

Borde, T. (2007). GemeindedolmetscherInnen in Berlin. Gut qualifiziert für ein neues Arbeitsfeld? In T. Borde \& N.-J. Albrecht (Eds.), Innovative Konzepte für Integration und Partizipation: Bedarfsanalyse zur interkulturellen Kommunikation in Institutionen und für Modelle neuer Arbeitsfelder (pp. 248-271).

Borde, T. (2018). Kommunikation und Sprache. Gynäkologische Endokrinologie, 16(1), 3-9. https://doi.org/10.1007/s10304-0170167-6.

Brandl, E. J., Schreiter, S., \& Schouler-Ocak, M. (2020). Are Trained Medical Interpreters Worth the Cost? A Review of the Current Literature on Cost and Cost-Effectiveness. Journal of Immigrant and Minority Health, 22(1), 175-181. https://doi.org/10.1007/ s10903-019-00915-4.

Brenne, S., Breckenkamp, J., Razum, O., David, M., \& Borde, T. (2013). Wie können Migrantinnen erreicht werden? Forschungsprozesse und erste Ergebnisse der Berliner Perinatalstudie. In E. Esen \& T. Borde (Eds.), DEUTSCHLAND UND DIE TÜRKEI - BAND II: Vol. 2. FORSCHEN, LEHREN Und ZUSAMMENARBEITEN in GESELLSCHAFT, GESUNDHEIT und BILDUNG (pp. 183198). Desen Ofset A. Ş.

David, M. (Ed.). (2017). Migration - Frauen - Gesundheit: Perspektiven im europäischen Kontext : [Dokumentation unseres zweiten
Symposiums vom 29. September 1999 in Berlin zum Thema „Migration - Frauen - Gesundheit im europäischen Kontext“ (3. Aufl.). Frankfurt/M.: Mabuse.

Destatis Statistisches Bundesamt. (2019). Geburten: Lebendgeborene nach Staatsangehörigkeit der Mutter. https://www.destatis.de/DE/ Themen/Gesellschaft-Umwelt/Bevoelkerung/Geburten/Tabellen/ lebendgeborene-staatsangehoerigkeit-laender.html

Divi, C., Koss, R. G., Schmaltz, S. P., \& Loeb, J. M. (2007). Language proficiency and adverse events in US hospitals: A pilot study. International Journal for Quality in Health Care: Journal of the International Society for Quality in Health Care, 19(2), 60-67. https://doi.org/10.1093/intqhe/mzl069.

Eickhorst, A. e. a. (2015). Die Prävalenzstudie „Kinder in Deutschland - KiD 0-3“ zur Erfassung von psychosozialen Belastungen und Frühen Hilfen in Familien mit 0-3-jährigen Kindern: Studiendesign und Analysepotential. Soziale Passagen, 7(2), S. 381-388.

Friebertshäuser, B., Richter, S., \& Boller, H. (2013). Theorie und Empirie im Forschungsprozess und die „Ethnographische Collage“ als Auswertungsstrategie. In B. Friebertshäuser, A. Langer, \& A. Prengel (Eds.), Handbuch qualitative Forschungsmethoden in der Erziehungswissenschaft (4th ed., pp. 379-396). Weinheim: Beltz Juventa.

Gläser, J., \& Laudel, G. (2010). Experteninterviews und qualitative Inhaltsanalyse als Instrumente rekonstruierender Untersuchungen (4. Auflage). Lehrbuch. Wiesbaden: Springer VS. http://d-nb. info/1002141753/04.

Goffman, E., Herkommer, H., \& Knoblauch, H. (2009). Interaktion im öffentlichen Raum. Frankfurt/M.: Campus.

Gün, A. K. (2018). Interkulturelle therapeutische Kompetenz: Möglichkeiten und Grenzen therapeutischen Handelns (1. Aufl.). Stuttgart: W. Kohlhammer.

Hadziabdic, E., \& Hjelm, K. (2014). Arabic-speaking migrants' experiences of the use of interpreters in healthcare: A qualitative explorative study. International Journal for Equity in Health, 13, 49. https://doi.org/10.1186/1475-9276-13-49.

Hollstein, B. (2006). Qualitative Methoden und Netzwerkanalysen. In B. Hollstein \& F. Straus (Eds.), Qualitative Netzwerkanalyse: Konzepte, Methoden, Anwendungen (1st ed., pp. 11-36). Wiesbaden: Springer VS..

Leanza, Y. (2005). Roles of community interpreters in pediatrics as seen by interpreters, physicians and researchers. Interpreting, 7(2), 167-192.

Ledyaikina, X., Uebelacker, J., \& Borde, T. (2007). Sprach- und Kulturmittlung in medizinischen und sozialen Einrichtungen aus der Sicht von MitarbeiterInnen. In T. Borde \& N.-J. Albrecht (Eds.), Innovative Konzepte für Integration und Partizipation: Bedarfsanalyse zur interkulturellen Kommunikation in Institutionen und für Modelle neuer Arbeitsfelder (pp. 100-119).

Mayring, P. (2016). Einführung in die qualitative Sozialforschung: Eine Anleitung zu Qualitativen Denken (6., überarbeitete Aufl.). Pädagogik. Weinheim: Beltz.

McKnight, P., Goodwin, L., \& Kenyon, S. (2019). A systematic review of asylum-seeking women's views and experiences of UK 
maternity care. Midwifery, 77, 16-23. https://doi.org/10.1016/j. midw.2019.06.007.

Meuser,M.,\&Nagel,U.(2013). Experteninterviews-wissenssoziologische Voraussetzungenund methodische Durchführung. In B. Friebertshäuser, A. Langer, \& A. Prengel (Eds.), Handbuch qualitative Forschungsmethoden in der Erziehungswissenschaft (4th ed., pp. 457-472). Weinheim: Beltz Juventa.

Origlia Ikhilor, P., Hasenberg, G., Kurth, E., Asefaw, F., PehlkeMilde, J., \& Cignacco, E. (2019). Communication barriers in maternity care of allophone migrants: Experiences of women, healthcare professionals, and intercultural interpreters. Journal of Advanced Nursing, 75(10), 2200-2210. https://doi.org/10.1111/ jan. 14093.

Razum, O., \& Spallek, J. (2015). Migration und Gesundheit. Public Health Forum, Band 23(2), 46-52.

RundUm. Ergebnisbroschüre: Transkulturelles Netzwerk zur Begleitung bei Schwangerschaft und Geburt. (2020). Online verfügbar https:// www.hs-gesundheit.de/fileadmin/user_upload/Aktuelles_Presse/ News-PDF/20200901RundUm_Ergebnisbericht.pdf.

SprInt Servicestelle. (2020). Das SprInt Netzwerk. https://www. sprachundintegrationsmittler.org/sprint-netzwerk/.

Sturman, N., Farley, R., Claudio, F., \& Avila, P. (2018). Improving the effectiveness of interpreted consultations: Australian interpreter, general practitioner and patient perspectives. Health \& Social Care in the Community, 26(2), e233-e240. https://doi.org/10.1111/ hsc. 12504 .

Wächter, M., \& Vanheiden, T. (2015). Sprachmittlung im Gesundheitswesen: Erhebung und einheitliche Beschreibung von Modellen der Sprachmittlung im Gesundheitswesen. Landesvereinigung für gesundheit und Akademie für Sozialmedizin Niedersachsen e. V. https://www.bikup.de/ wp-content/uploads/2016/07/Studie_Sprachmittlung-imGesundheitswesen.pdf.

Wolter, H., Grieger, D., \& Wesselman, E. (2007). Einleitung: Gute -Praxis-Beispiele für Gesundheit und Integration. In Gesundheit und Integration: Ein Handbuch für Modelle guter Praxis (3rd ed., pp. 9-16). Beauftragte der Bundesregierung für Migration Flüchtlinge und Integration. 